\title{
Long-range Mobile Technology is the Future of Providing Bespoke Surgical Interventions
}

\author{
Rupali D. Shah • Thomas I. Lemon
}

Received: 25 February 2013 /Accepted: 27 February 2013 /Published online: 13 March 2013

(C) Association of Surgeons of India 2013

Dear Editor,

This correspondence is in regard to Satish Shukla and Chiranjiva Khandelwal "Surgery—Passion, Proficiency, Perfection and Profession" Indian J Surg doi:10.1007/s12262013-0884-6.

Firstly, we would like to thank the authors for such an interesting and relevant article. Additionally, we appreciate the brief history of medicine provided, in particular the impact Sushruta had on surgery and the history of the primitive sort of analgesia (hemlock juice). As aspiring surgeons, we find these facts fascinating.

The specialisation of surgery is a necessary but somewhat unfortunate situation. On one hand, it is a necessity to have surgeons who can perform a very specialised operation (cardiothoracic) and know the ins and out of it, and, on the other hand, it would be administratively and economically useful if one surgeon had broad-ranging expertise spanning specialities. We argue that specialisation is of more use within urban settings as opposed to rural settings where the nearest hospital may be hundreds of miles away. Within the UK, one could argue that due to increasing numbers of air ambulances and other resources, specialised surgeons are able to serve wider areas. However, many services such as Plastics are still limited to a few tertiary centres.

In an ideal world, every hospital would have every speciality represented, or surgeons would be superhumans who could practice with the competence they pride themselves on across multiple specialities. Alas, this is not the case, but with the advent of improved mobile technologies, perhaps specialist surgeons of the future will be able to operate over long-range bluetooth using robotics.

R. D. Shah · T. I. Lemon $(\bowtie)$

School of Medicine, Cardiff University, Cardiff, UK

e-mail: lemonti@cf.ac.uk 